Methods Thirty three clinically active pts with LN underwent glomerular filtration rate (GFR) clearance study by using radionucleotide compound Tc-99 m diethylamine-pentoacetic acid (DTPA). All pts had definite clinical evidence of active LN, including urinary and immunological abnormalities. Twenty of pts underwent renal biopsies (66\%). GFR was studied in 30 female and 3 male pts (average 39 years and duration of disease average 5 years) in two occasions over an interval of 3 to 6 months during treatment with cotricosteroids (Cs) and cytotoxic drugs $(\mathrm{Ct})$.

Results GFR-DTPA clearance was in range from 11 to $157 \mathrm{ml} /$ min, mean $87.42 \mathrm{ml} / \mathrm{min}$ (normal $>100 \mathrm{ml} / \mathrm{min}$ ), in repeated measurements it was in range from 13 to $147 \mathrm{ml} / \mathrm{min}$, mean GFR (94.33) was slightly but not significantly better. We divided pts into four groups. First group of 12 pts had values in normal range, second group of $11 \mathrm{pts}$ showed an improvement in GFR from mean 64.33 to $95.50 \mathrm{ml} / \mathrm{min}$. Mean GFR second values from third group of 6 pts (18\%) were as low as in first measurements (mean 51.83), and a fall in GFR (mean 106 to $70.25 \mathrm{ml} /$ $\min$ ) was notified only in 4 pts $(12 \%)$ as a result of deterioration of glomerular function.

Conclusion During the undergoing treatment over an interval of 3 to 6 month we kept GFR in pts with $\mathrm{LN}$ in normal range at 12 pts $(37 \%)$. Average GFR increased significantly $(\mathrm{p}<0.05)$ at 11 pts (33\%). The treatment $\mathrm{LN}$ with Cs and Ct is useful for saving renal function, and could be able to prevent the progression $\mathrm{LN}$ to renal failure.

\section{FRI0107 TOXICITY OF ANTIMALARIALS IN THE TREATMENT OF PORTUGUESE PATIENTS WITH SYSTEMIC LUPUS ERYTHEMATOSUS}

H Canhão, JE Fonseca, JC Teixeira Costa, JA Pereira Silva, M Viana Queiroz. Rheumatology Unit, Santa Maria Hospital, Lisbon, Portugal

\subsection{6/annrheumdis-2001.142}

Background Chloroquine and hydroxychloroquine are drugs widely used in the treatment of systemic lupus erythematosus (SLE).

Objectives The aim of our study was to assess the toxicity of antimalarials in the treatment of SLE Portuguese patients.

Methods 234 outpatients satisfying the 1982 revised ARA criteria for SLE and regularly followed in our Rheumatology Unit were evaluated. $222(94,9 \%)$ were females and $12(5,1 \%)$ were males. The mean age of SLE onset was 30,1 years. The mean duration of follow-up was 5,5 years.

Results $157(67,1 \%)$ patients were medicated with antimalarials, $59(37,6 \%)$ with chloroquine in a daily dose of $3-5 \mathrm{mg} / \mathrm{kg}$ and $98(62,4 \%)$ with hydroxychloroquine in a daily dose of $5-7 \mathrm{mg} /$ kg. $77(32,9 \%)$ patients were never treated with chloroquine or hydroxychloroquine. In the chloroquine group, $28(47,5 \%)$ patients were still on therapy and in $31(52,5 \%)$ patients the chloroquine was stopped. In the hydroxychloroquine group 64 $(65,3 \%)$ patients remain on therapy and in $34(34,7 \%)$ patients the drug was suspended. The reasons to stop chloroquine were ocular toxicity in $20(64,5 \%)$ patients, gastrointestinal intolerance in $5(16,1 \%)$, cutaneous rash in $2(6,4 \%)$, patient will in 2 $(6,4 \%)$ and medical advice but without adverse effects in 2 $(6,4 \%)$. The reasons to suspend hydroxychloroquine were ocular toxicity in $15(44,1 \%)$ patients, patient will in $6(17,6 \%)$, cutaneous rash in $3(8,8 \%)$, medical advice but without adverse effects in $3(8,8 \%)$, pregnancy in $3(8,8 \%)$, death not related with the antimalarial in 2 (5,9\%), headache and dizziness in 1 $(2,9 \%)$, gastrointestinal intolerance in 1 (2,9\%).

Conclusion In our patients the antimalarial discontinuation was frequent and occurred in $65(41,4 \%)$ out of the 157 treated patients. It occurred more often in the chloroquine group $(52,5 \%)$ as compared to the hydroxychloroquine $(34,7 \%)$ group. Most of the adverse effects reported were mild. Ocular toxicity was the most frequent adverse effect implicated in the drug discontinuation and it was reported in $35(22,3 \%)$ of the 157 treated patients. Chloroquine induced more ocular toxicity as compared to hydroxychloroquine (64,5\% vs 44,1\%). Most of the patients that have stopped the drug because of the abnormalities detected in the ophthalmologic evaluation had no clinical symptoms. There were no blindness reports. The great number of ocular toxicity cases detected could be the result of the use of high sensitive tools in the ophthalmologic evaluation such as computerised evoked potentials or fluoresceinic angiography. In addition, other factors such as sun exposure and low body mass might have contributed to a higher rate of ocular toxicity as compared to Northern European reports. In the 157 patients group there were no reports of muscle, neurological, liver, renal or haematological abnormalities due to the antimalarial therapy.

The antimalarials are useful and relatively safe drugs in the treatment of SLE. Hydroxychloroquine seems to be less toxic than chloroquine. The need of a periodic ophthalmologic evaluation once or twice yearly was supported by our study.

\section{FRI0108 RETINAL CHANGES AND ACTIVITY IN SYSTEMIC LUPUS ERYTHEMATOSUS}

${ }^{1} \mathrm{Z}$ Hrncir, ${ }^{2} \mathrm{E}$ Rencova, ${ }^{1} \mathrm{~T}$ Soukup, ${ }^{1} \mathrm{P}$ Bradna, ${ }^{1} \mathrm{Z}$ Dvorak. '2nd Department of Medicine; ${ }^{2}$ Department of Ophthalmology, University Hospital, Hradec Kralove, Czech Republic

\subsection{6/annrheumdis-2001.143}

Background The incidence of retinopathy in systemic lupus erythematosus (SLE) and its relation to other clinical and laboratory manifestations of SLE are under debate. ${ }^{1}$ Great importance is placed on retinopathy in activity criteria according to the scoring system of SLEDAI. ${ }^{2}$

Objectives The goal of the monocenter cross sectional study from the East Bohemian region is to clarify the problem according to the incidence of retinal changes of SLE, and evaluation of the disease activity in SLE according to the descriptors of the SLEDAI $^{2}$ in patients with and without retinopathy.

Methods The group under study consists of 60 SLE patients according to ACR classification (1982, updated 1997). One expert ophthalmologist examined the ocular fundi for the presence of retinal changes fulfilling the definition of the SLEDAI descriptor for visual disturbance, but without any information about other SLEDAI descriptors. Data obtained in SLE patients with and without retinopathy were statistically processed using Wilcoxon's test and Fisher's exact test.

Results Retinopathy according to the SLEDAI definition was present in 9 (15\%), and absent in 51 (85\%) SLE patients. The values of the SLEDAI score in patients with retinopathy were significantly higher than in patients without retinopathy $(\mathrm{p}<$ 0.001). In SLE with retinopathy two other SLEDAI descriptors frequency was significantly more than in patients without retinopathy: arthritis $(\mathrm{p}<0.035)$ and increased DNA binding $(\mathrm{p}<$ 0.038).

Conclusion The incidence of retinopathy according to the SLEDAI definition in $15 \%(9 / 60)$ of SLE under study, and 
significantly higher values of the SLEDAI score in SLE subgroup with retinopathy were found. Data obtained also demonstrated that retinal changes of SLE may be significantly more frequent in SLE with arthritis and with increased DNA binding.

\section{REFERENCES}

1 Ushiyama 0, et al. Ann Rheum Dis. 2000:705-8

2 Bombardier C, et al. Arthritis Rheum. 1992:35:630-40

\section{FRI0109 SURVIVAL AND PREDICTOR VARIABLES FOR MORTALITY IN SYSTEMIC LUPUS ERYTHEMATOSUS}

S Appenzeller, LT Costallat. Internal Medicine, State University of Campinas-UNICAMP, Campinas, Brazil

\subsection{6/annrheumdis-2001.144}

Background Survival of systemic lupus erythematosus (SLE) patients has improved significantly over time because of recognition of mild disease and better approaches to therapy. The identification of predictor variables for mortality is necessary to identify subgroups at greater risks to die and who need more aggressive therapy.

Objectives To analyse survival and mortality risks of various clinical variables in a cohort of SLE patients, followed prospectively in a single centre.

Methods The survival of a cohort of 509 SLE patients, SLE followed over a 20-year period according to a standard protocol was studied using the Kaplan Meier lifetable analysis methods. Univariate associations between clinical and laboratory informations and drugs used were calculated. The Cox proportional hazard regression model was used to estimate risk ratio of death and to examine the simultaneous effects of multiple prognostic factors.

Results Over the duration of follow-up of 20 years, 58 (11.4\%) died. The survival rate was $97 \%$ at 1 year, $90 \%$ at 5 years, $81 \%$ at 10 years and $75 \%$ at 20 years. Age $<16$ years at diagnosis, hypertension, weight loss, nephritis, ocular involvement, the presence of anti DNA antibodies, the absence of artralgia/arthritis and frequent infections were significantly associated with reduced survival in univariate analysis. Using multivariate analysis, hypertension (OR 2.2, 95\% CI 1.1-4.5), age $<16$ years at diagnosis (OR 2.6, 95\% CI 1.4-5.0), ocular involvement (OR 4.9 , 95\% CI 1.9-12.8), treatment without antimalarial drugs (OR 2.8, 95\% CI 0.15-0.53) and frequent infections (OR 2.3, 95\% CI 1.3-4.1) were independent risk factors associated with increased mortality.

Conclusion Hypertension, age $<16$ years at diagnosis, ocular involvement, treatment without antimalarial drugs and frequent infections were independent risk factors associated with increased mortality.

\section{REFERENCES}

1 Uramoto, Kristine M, Michet CJJ, et al. Trends in the incidence of mortality of SLE. Arthritis Rheum 1999:42(1)

2 Petri M. Hopkins Lupus Cohort. Rheum Dis Clin N Am. 2000;26(2)

\section{FRI0110 BOWEL PERFORATION IN SYSTEMIC LUPUS ERITHEMATOSUS}

${ }^{1} \mathrm{ME}$ Pombo, ${ }^{2} \mathrm{~L}$ Diez, ${ }^{2} \mathrm{Jl}$ Bernardino, ${ }^{2} \mathrm{~F}$ García, ${ }^{2} \mathrm{M}$ Mora, ${ }^{2} \mathrm{P}$ Lavilla, ${ }^{2} \mathrm{~A}$ Gil. ${ }^{1}$ Rheumatology Service; ${ }^{2}$ Internal Medicine Service, Hospital Universitario La Paz, Madrid, Spain

\section{Background}

Objectives Bowel perforation is a rare but serious complication in SLE. Although several processes not related to SLE (diverticulitis, tumours) may be the cause of this complication, it has been previously considered to be associated to small-vessel vasculitis in active lupus disease. Nowadays, a vascular damage induced by antiphospholipid antibodies (aPL) and steroids seems to play an important role.

Methods Seven patients with intestinal perforation were observed in 220 SLE patients during a period of 26 years (1974-2000). In all cases, the diagnosis of intestinal perforation was confirmed by surgery or autopsy and pathological examination.

Results All patients were females, with a mean age at the diagnoses of bowel perforation of 45 years, and a mean time of SLE evolution of 13 years. The site of perforation was in the colon in 4 patients and in the small bowel in the other 3. Six patients had lupus nephropathy, one of them with renal transplantation. Central nervous system and cutaneous involvement was observed in 4 patients and Sjögren's syndrome, Libman-Sachs endocarditis and haemolytic anaemia in two patients. aPL were present in 5 patients. All patients were on steroid therapy at the moment of the intestinal perforation. Abdominal pain was present in all cases but the mean time of diagnosis delay was 11 days. Two patients died from peritonitis and septic shock. A local cause of perforation was found in four patients: diverticulitis (3) and adenocarcinoma of the rectum (1). Necrotizing vasculitis was not observed in any case of pathological examinations.

Conclusion Bowel perforation, although uncommon in SLE, has to be taken into account as a differential diagnosis in a patient with abdominal pain. Disease severity, antiphospholipid antibodies and previous steroid therapy are important risk factors for this complication.

\section{FRI0111 PULSE METHYLPREDNISOLONE AND CYCLOPHOSPHAMIDE THERAPY IN THREE SYSTEMIC LUPUS ERYTHEMATOSUS PATIENTS WITH BRONCHIOLITIS OBLITERANS ORGANISING PNEUMONIA AS THE INITIAL MANIFESTATION}

JC Tseng, HH Cheng, RJ Hu, LY Lu. Allergy, Immunology and Rheumatology, Veterans General Hospital- Kaohsiung, Kaohsiung, Taiwan, R.O.C

\subsection{6/annrheumdis-2001.146}

Background Diverse pleuropulmonary manifestations are common in patients with systemic lupus erythematosus (SLE). Bronchilolitis obliterans organising pneumonia (BOOP) is a pathologic entity characterised by the formation of fibrous tissue plugs within terminal bronchioles and alveolar ducts. Although BOOP has been associated with several connective tissue disorders, there are rare reports of BOOP in patients of SLE as the initial manifestation.

Objectives We describe three SLE patients with open lung biopsy-proved BOOP as the initial manifestation. All three cases presented with fever, cough and dyspnea. Chest radiographs and CT scan of lung revealed bilateral pulmonary infiltrates. BOOP was confirmed by open lung-biopsy. Pulmonary vasculitis was also demonstrated in lung histopathology in one case.

Methods

Results The pulmonary lesions and respiratory illnesses responsed well to pulse intravenous methylprednisolone and cyclophosphamide therapy. 